\title{
Whole blood assays for interferon- $\gamma$ : practicalities and potential for use as diagnostic tests in the field
}

\author{
H. M. DOCKRELL*, G. F. BLACK**, R. E. WEIR* \& \\ P. E. M. FINE* \\ *Department of Infectious and Tropical Diseases, London School of \\ Hygiene \& Tropical Medicine, London, UK and **Karonga \\ Prevention Study, Chilumba, Malawi
}

\begin{abstract}
Summary Recent years have seen the introduction of a number of whole-blood assays, in which unseparated heparinized blood is stimulated with antigen either overnight or for as long as 6 days, and cytokine production is measured in the plasma or supernatant. These assays have potential for use in the field as immunodiagnostic assays, as they require only a small blood sample and basic laboratory facilities. Use of these assays in a large study of the immunological effects of BCG vaccination in Malawi has shown that the diluted blood, 6-day whole-blood assay is robust, and can be used to assess T-cell responses to both crude and recombinant antigens. If used with antigens specific to Mycobacterium leprae, these assays could be used to measure exposure to $M$. leprae within communities or populations, or to aid the early diagnosis of leprosy.
\end{abstract}

Whole blood assays, in which heparinized whole blood, rather than gradient-separated, peripheral blood mononuclear cells (PBMC), is used to study cell function, can be employed to measure both lymphocyte proliferation and cytokine production. As the blood is simply diluted and placed in culture with the appropriate stimulus, the assays are far simpler and quicker to set up than conventional assays using PBMC, and can be performed in laboratories with only basic facilities. This means that they can be used in a community, to study the immune response to exposure to Mycobacterium leprae.

Whole-blood assays are being used increasingly for research or diagnosis. ${ }^{1}$ In these assays, undiluted blood can be incubated with antigen overnight, ${ }^{2,3}$ or blood, diluted 1:10 or $1: 5$, can be incubated for 5-6 days. ${ }^{4-6}$ We have been using heparinized blood diluted 1:10, which is incubated with antigens or mitogens at $37^{\circ} \mathrm{C}$ in an atmosphere of $5 \% \mathrm{CO}_{2}$; supernatants are collected after 5-6 days to measure T-cell-derived cytokines. Our initial studies in Nepal showed that the IFN- $\gamma$ response to fractionated leprosy antigens correlated with position in the leprosy spectrum. ${ }^{7}$

We are now employing this method in a large, field-based study of the immune response to BCG vaccination in Malawi, where BCG has previously been shown to provide some protection against leprosy, but not against pulmonary tuberculosis; ${ }^{8}$ a parallel study is being performed in the United Kingdom. In Malawi, testing of skin-test sensitivity and cytokine production to mycobacterial antigens was performed in 635 individuals, who were then randomly allocated, so that two-thirds received BCG vaccination and one-third placebo. The results of pre-vaccination testing of the IFN- $\gamma$ responses are now available, permitting us to 
evaluate the practicalities of using such whole-blood assays on a broad scale, and to determine whether they are sufficiently robust for field use.

One problem with performing such assays over time, and under field conditions, in which the ambient temperature in the laboratory may vary considerably during the year, is to control for the performance and reproducibility of the cytokine ELISAs. We have used aliquots of a positive control supernatant, obtained by incubating bulk cultures of heparinized whole blood diluted 1:10 with phytohaemagglutinin, a mitogen, for 3 days, as a biological control that is included on each plate; this has produced a coefficient of variation of $18 \%$. Such supernatants can also be exchanged between laboratories, to ensure comparability of the quantities of cytokine measured. Comparison of the standard curves run on each ELISA plate also reveals changes in sensitivity of the assay. The reproducibility of whole-blood cytokine responses over time within the same individual is also an issue. In a study in the UK and Nepal, the responses of individual controls and patients tested monthly over 6 months proved consistent. $^{9}$

Responses to a panel of purified protein derivatives (PPDs) from a range of mycobacterial species have been used in the Malawi study to assess exposure to pathogenic and environmental mycobacteria. Using the antigens at a concentration of $5 \mu \mathrm{g}$ per $\mathrm{ml}$, and a cut-off of $62 \mathrm{pg}$ per $\mathrm{ml}$ of IFN- $\gamma$ to define a responder, responders and non-responders were detected to all of the antigens used. Overall, frequency distributions indicated that, in Malawi, exposure to some of the environmental mycobacteria such as $M$. avium may be greater than that to M. tuberculosis (Black, Fine and Dockrell, unpublished results).

It was also important to assess whether assays performed on diluted whole blood are sufficiently sensitive to allow detection of the responses to individual recombinant antigens, particularly as cultures of blood diluted 1:10 contain only approximately one-quarter of the mononuclear cells used in standard PBMC assays. A panel of recombinant antigens from $M$. tuberculosis, M. leprae, and $M$. bovis has been used. Results indicate that, although the proportion of responders to individual antigens, and the median IFN- $\gamma$ produced are lower than those obtained with crude PPD preparations, the assays are sufficiently sensitive to detect responses to the recombinant antigens, and to reveal varying responses to those derived from the different mycobacteria (Black, Fine and Dockrell, unpublished results).

Another question of interest is whether the results of such IFN- $\gamma$ assays parallel the results obtained by skin testing; if so, these assays could be used to screen leprosy antigens or fractions for those of diagnostic potential, prior to their formulation as skin-test reagents. Alternatively, whole-blood assays could be used instead of a skin test; although a small blood sample is required, a repeat visit to read the skin test is not required. Whole blood tests have been proposed as an alternative to skin testing for the diagnosis of tuberculosis. ${ }^{2,3}$ When the IFN- $\gamma$ response induced in vitro by PPD (RT48, Statens Serum Institut, Copenhagen) was compared to the induration measured $48-72 \mathrm{~h}$ after skin testing with 2 TU of PPD RT23, a very high correlation was obtained between the median IFN- $\gamma$-response and induration, ${ }^{10}$ suggesting that these responses are correlated in the majority of individuals, although there are exceptions - individuals who respond in one test or the other, but not in both.

In conclusion, whole blood assays, using heparinized blood diluted 1:10, and stimulated with antigen for 6 days, allow measurement of cytokines such as IFN- $\gamma$ in large-scale field studies. The ELISA method for detection of cytokine is robust, and the inclusion of control supernatants allows assay variability to be monitored. The responses in healthy individuals appear stable over time. These assays allow responses to both crude antigens such as PPDs and individual recombinant antigens to be evaluated. The 6-day IFN- $\gamma$ response to 
M. tuberculosis PPD shows a strong association with induration measured by skin testing to the same antigen. If used with $M$. leprae-specific antigens, such whole blood tests have potential as field tools with which to monitor exposure to $M$. leprae within communities, or as a diagnostic test to aid the early diagnosis of leprosy.

\section{Acknowledgements}

Development of the whole-blood method was funded by a $\mathrm{PhD}$ grant to $\mathrm{Dr} \mathrm{R}$. Weir from the Hospitals and Homes of St Giles, UK. The BCG vaccination study in Malawi is funded by the Wellcome Trust, and the parallel study in the UK by LEPRA. We thank Lifted Sichali and Lorren Mwangulu for collecting the blood samples, and Steven Chaguluka for technical support. We also thank the people of Karonga District for their co-operation.

\section{References}

${ }^{1}$ Dockrell HM, Weir RE. Whole blood cytokine assays - a new generation of diagnostic tests for tuberculosis? Int J Tuberc Lung Dis, 1998; 2: 441-442.

2 Desem N, Jones SL. Development of a human gamma interferon enzyme immunoassay and comparison with tuberculin skin testing for detection of Mycobacterium tuberculosis infection. Clin Diagn Lab Immunol, 1998; 5: 531-536.

3 Streeton JA, Desem N, Jones SL. Sensitivity and specificity of a gamma interferon blood test for tuberculosis infection. Int J Tuberc Lung Dis, 1998; 2: 443-450.

${ }^{4}$ Frankenburg S. A simplified microtechnique for measuring human lymphocyte proliferation after stimulation with mitogen and specific antigen. J Immunol Meth, 1988, 112: 177-182.

5 Weir RE, Morgan AR, Britton WJ et al. Development of a whole blood assay to measure T cell responses to leprosy: a new tool for immuno-epidemiological field studies of leprosy immunity. J Immunol Meth, 1994; 176: 93-101.

${ }^{6}$ Elliott AM, Hurst TJ, Belyeku MN et al. The immune response to Mycobacterium tuberculosis in HIV-infected and uninfected adults in Uganda: application of a whole blood assay in an epidemiological study. Int J Tuberc Lung Dis, 1999; 3: 239-247.

7 Weir RE, Brennan PJ, Butlin CR, Dockrell HM. Use of a whole bloodassay to evaluate in vitro T cell responses to new leprosy skin test antigens in leprosy patients and healthy subjects. Clin Exp Immunol, 1999; 116: 263-269.

8 Karonga Prevention Trial Group. Randomised controlled trial of single BCG, repeated BCG, or combined BCG and killed Mycobacterium le prae vaccine for prevention of leprosy and tuberculosis in Malawi. Lancet, 1996; 348: 17-24.

9 Weir RE, Butlin CR, Neupane KD et al. Use of a whole blood assay to monitor the immune response to mycobacterial antigens in leprosy patients: a predictor for type 1 reaction onset? Lepr Rev, 1998; 69: $279-293$.

10 Black GF, Fine PEM, Wamdorff DK et al. Patterns of IFN- $\gamma$ and skin test responsiveness to Mycobacterium tuberculosis PPD in healthy, non-BCG-vaccinated young adults in northern Malawi. Submitted for publication, 2000. 